\title{
Práctica quirúrgica durante la pandemia por COVID-19: revisión de literatura
}

\author{
Surgical practice during the COVID-19 pandemic: literature review \\ María Cristina Ornelas Flores, ${ }^{*}$ María Fernanda Parada Pérez, ${ }^{*}$ Mónica León González, ${ }^{*}$ \\ Florencia Lucero Serrano, ${ }^{*}$ Carlos Guillermo Mondragón Salgado, ${ }^{*}$ Leopoldo Castañeda Martínez ${ }^{\ddagger}$
}

\section{RESUMEN}

Objetivo: El propósito de este artículo es proveer información actualizada que ayude al cirujano a llevar su práctica quirúrgica con seguridad durante la pandemia por COVID-19. Método: Se realizó una revisión de la literatura actual disponible con un análisis crítico de los artículos, páginas de Internet de organizaciones mundiales oficiales y manuales de manejo estandarizado. Resultados: Se logró crear una guía de indicaciones quirúrgicas abarcando múltiples áreas y especialidades de interés que ayudarán al cirujano a llevar su práctica quirúrgica con seguridad durante y después de la pandemia. Conclusión: La práctica quirúrgica como la conocemos ha cambiado radicalmente a raíz de la pandemia. La información recabada de la literatura disponible únicamente realiza recomendaciones que surgen de datos ya conocidos y de opiniones de expertos, por lo que esta guía de recomendaciones no debe ser seguida rigurosamente, se debe aplicar el criterio del cirujano en cada situación específica e individualizar cada caso. Nuevos hallazgos científicos hacen que la información cambie rápidamente y, por esto, es probable que las estrategias y medidas descritas en este artículo cambien conforme vamos adquiriendo conocimiento sobre el COVID-19.

Palabras clave: Coronavirus, COVID-19, SARS-CoV-2, pandemia, cirugía, laparoscopía.

\section{ABSTRACT}

Objective: The purpose of this article is to provide updated information to help the surgeon execute his surgical practice safely during the COVID-19 pandemic. Method: A review of the current available literature was carried out with a critical analysis of the articles, internet pages of official world organizations and standardized management manuals. Results: A guide of surgical indications was created including multiple areas and surgical specialties of interest that will help the surgeon follow through during and after the pandemic. Conclusion: Surgical practice as we know it has radically changed as a result of the pandemic. The information collected from the available literature makes recommendations that emerge from known data and experts opinions. As a result, this guide of recommendations should not be followed rigorously, the surgeon's criteria should be applied in each specific situation and individualizing each case. New scientific findings are updating the information rapidly, and therefore, the strategies and measures described in this article are likely to change as we gain knowledge about COVID-19.

Keywords: Coronavirus, COVID-19, SARS-CoV-2, pandemic surgery, laparoscopy.

\section{INTRODUCCIÓN}

\section{Abreviaturas:}

COVID-19: Coronavirus Disease 2019

SARS-CoV-2: Severe Acute Respiratory Syndrome Coronavirus 2.

ACS: Colegio Americano de Cirujanos.

TC: Tomografía computarizada.

PCR: Proteína C reactiva.

UTI: Unidad de Terapia Intensiva.

EPP: Equipo de protección personal.

VMI: Ventilación mecánica invasiva.

CDC: Centro para Control de Enfermedades.
La pandemia por COVID-19 ha afectado al mundo entero y ha obligado a los países a adaptar su sistema de salud para poder manejar el incremento de flujo de pacientes infectados. ${ }^{1}$ El COVID-19 es una infección respiratoria aguda causada por un virus perteneciente a la familia coronavirus- $\beta$, Ilamado SARS-CoV-2.1,2 A raíz de la

* Médico residente Cirugía General. ₹ Cirujano General. Centro Médico ABC, Ciudad de México, México.

Recibido para publicación: 04/05/2020. Aceptado: 16/05/2020.

Correspondencia: Leopoldo Castañeda Martínez. Sur 136 Núm. 116, consultorio 213 Torre Mackenzie, Col. Las Américas, Alcaldía Álvaro Obregón. 01120, Ciudad de México, México. Tel: 55 166942. E-mail: leopoldocastaneda@gmail.com

Citar como: Ornelas FMC, Parada PMF, León GM, Serrano FL, Mondragón SCG, Castañeda ML. Práctica quirúrgica durante la pandemia por COVID-19: revisión de literatura. Rev Mex Cir Endoscop. 2020; 21 (1): 41-53. https://dx.doi.org/10.35366/97613 
pandemia, la práctica quirúrgica como la conocemos ha cambiado radicalmente. Nos hemos visto en la necesidad de desviar los recursos médicos y humanos antes destinados a otras patologías para tratar a los pacientes infectados con SARS-CoV-2. Cada sistema de salud, hospital y cirujano ha tenido que revisar sus procedimientos programados con un plan para minimizar, posponer o cancelar todos aquellos procedimientos electivos o invasivos no urgentes. A pesar de que el flujo de información sobre el COVID-19 va en aumento, es poco lo que sabemos sobre el manejo quirúrgico seguro de los pacientes infectados y sobre la prevención en pacientes sanos.

El propósito de este artículo es proveer información actualizada que ayude al cirujano a llevar su práctica quirúrgica con seguridad durante la pandemia por COVID-19, tanto para él y su equipo quirúrgico, como para el paciente y su entorno, todo esto mientras se optimizan los recursos disponibles.

\section{MÉTODO}

Se realizó una revisión de literatura actualizada con un análisis crítico de los artículos, páginas de Internet de organizaciones mundiales oficiales y manuales de manejo. Se utilizó PubMed como sitio de búsqueda aplicando palabras clave como: coronavirus, COVID-19, cirugía, cirugía electiva, cirugía laparoscópica.

\section{Protocolo preoperatorio}

Los pacientes sanos que no representan una urgencia quirúrgica pero que ameritan vigilancia hospitalaria en el Servicio de Cirugía deberán contar con proteína $C$ reactiva (PCR) y tomografía computarizada (TC) de tórax previo ingreso. ${ }^{3,4}$ Todos los pacientes deberán tener una prueba de PCR para SARS-CoV-2 sin que esto retrase el manejo de urgencia en pacientes críticos con requerimiento de procedimientos quirúrgicos. ${ }^{5}$ En caso de retraso en la prueba y de disponibilidad en servicios de imagen, se podrá completar la valoración preoperatoria con una radiografía o TC de tórax. Los pacientes en los que se descartó COVID-19 deben trasladarse a quirófano de preferencia en una ruta diferente a la de los pacientes COVID-19 positivos y se deberán proteger mínimo con una mascarilla quirúrgica. ${ }^{6}$

Pacientes con síntomas sugestivos de COVID-19 que se presenten en urgencias con requerimientos quirúrgicos de atención inmediata deberán ser tratados como casos sospechosos hasta demostrar lo contrario y ser valorados con una radiografía, ultrasonido o TC de tórax en busca de patrones característicos de la infección..$^{5,6}$ El traslado de pacientes infectados o sospechosos a quirófano deberá ser planeado y coordinado con el Servicio de Seguridad del hospital, para asegurar bloqueo de pasillos y elevadores. Los pacientes se acompañan de personal de enfermería utilizando equipo de protección personal (EPP). ${ }^{7} \mathrm{Al}$ terminar el traslado, la ruta utilizada para su movilización deberá ser desinfectada con una solución de cloro en una concentración $>0.1 \%$ con un tiempo de contacto a superficie de mínimo 1 minuto. ${ }^{6}$ Se deberá designar una sala de operaciones específica para pacientes COVID-19 positivos. Esta sala debe tener presión negativa y de preferencia debe ser la más cercana a la entrada de quirófanos para agilizar protocolos. ${ }^{6,8}$

\section{Medidas de protección en el quirófano}

El EPP es siempre la principal prioridad durante cualquier procedimiento. Todo paciente sometido a un procedimiento quirúrgico debe ser tratado como un caso sospechoso hasta que se compruebe lo contrario. ${ }^{6}$ Según la CDC (Centro para Control de Enfermedades) es indispensable realizar lavado de manos antes y después de cada cirugía. ${ }^{9}$ Para el EPP dentro de quirófano se debe hacer uso de máscara protectora N95 o respiradores que cuenten con un adecuado nivel de protección. Las mascarillas deben ser retiradas posterior a cada procedimiento quirúrgico acorde a las indicaciones de cada institución. ${ }^{6}$ Se recomienda que en todo procedimiento realizado en el cual haya o no riesgo de aerosolización se utilice máscara N95, protección ocular, gorro y botas protectoras desechables, bata impermeable y doble par de guantes. En pacientes con prueba de PCR negativa y estudio de imagen normal se puede considerar el uso del equipo de protección convencional. ${ }^{6}$

Se debe estructurar al equipo de trabajo en el quirófano, teniendo siempre al personal mínimo indispensable para llevar a cabo el procedimiento. Los observadores están prohibidos en cualquier cirugía. Se recomienda que sólo el equipo de anestesiología se encuentre dentro del quirófano durante la intubación por el riesgo de aerosolización. ${ }^{6,9}$ Los procedimientos con invasión a la vía aérea representan riesgo de aerosolización, algunos ejemplos son: la intubación orotraqueal, aspiración de fluidos orales, realización de traqueostomía, colocación de sonda nasogástrica y los procedimientos endoscópicos. ${ }^{9}$

\section{Desinfección del quirófano y manejo de residuos}

El personal que sale del quirófano debe retirar su bata y guantes dentro del quirófano y realizar lavado de manos. Se requiere otorgar una hora como tiempo mínimo entre cirugías para el traslado fuera de quirófano del paciente y la descontaminación de la sala. Todo el material des- 
echable no utilizado se asumirá como contaminado y se desechará. ${ }^{7}$ Los materiales reusables deberán valorarse de acuerdo a las posibilidades de infección de cada paciente y serán descontaminados, lavados o esterilizados. Los aparatos electromédicos y todas las superficies se deben limpiar con una solución clorada, lavados, secados y desinfectados con otra solución clorada concentrada a $>0.1 \%$ y dejar en contacto con superficies por más de un minuto. Posterior a esto, el quirófano puede descontaminarse con un vaporizador de peróxido de hidrógeno o por irradiación ultravioleta. ${ }^{7,10}$ Durante la desinfección de quirófano, el personal de limpieza deberá utilizar sólo materiales de uso único, los cuales deberán ser desechados inmediatamente en los contenedores designados, para luego ser cerrados y sellados para su transporte al punto de colección.

\section{Estadificación de riesgo e indicación quirúrgica}

Es importante clasificar los procedimientos quirúrgicos conforme al tiempo que podemos esperar para realizarlo (Tabla 1), dependiendo de la urgencia y el riesgo que este implica en el paciente al ser retrasado. ${ }^{11}$ El Colegio Americano de Cirujanos (ACS) publicó recientemente la escala MeNTS (Medically-Necessary, Time-Sensitive), la cual funciona como sistema de categorización de riesgo quirúrgico

Tabla 1: Tiempos para atención de procedimientos quirúrgicos.

\begin{tabular}{|c|c|c|}
\hline Indicación & Tiempo & Procedimientos \\
\hline Emergencia & $<1$ hora & $\begin{array}{l}\text { - Patologías que pongan en riesgo la vida } \\
\text { - Hemorragias agudas o choque } \\
\text { - Oclusión o perforación intestinal } \\
\text { - Sepsis abdominal } \\
\text { - Peritonitis } \\
\text { - Fascitis necrotizante } \\
\text { - Síndrome compartimental } \\
\text { - Disección aórtica } \\
\text { - Aneurisma aórtico roto } \\
\text { - Lesión vascular aguda } \\
\text { - Hernias estranguladas }\end{array}$ \\
\hline Urgencia & $<24$ horas & $\begin{array}{l}\text { - Infección quirúrgica } \\
\text { - Apendicitis aguda } \\
\text { - Colelitiasis aguda } \\
\text { - Artritis séptica } \\
\text { - Lesión medular } \\
\text { - Hemorragia pélvica } \\
\text { - Fracturas expuestas } \\
\text { - Quemaduras }\end{array}$ \\
\hline Electiva urgente & $<2$ semanas & $\begin{array}{l}\text { - Aneurisma cerebral } \\
\text { - Accesos vasculares } \\
\text { - Procedimientos cardiotorácicos y cardiovasculares } \\
\text { - Fracturas cerradas } \\
\text { - Fractura de columna } \\
\text { - Injertos de piel y colgajos } \\
\text { - Colelitiasis crónica }\end{array}$ \\
\hline Electiva no urgente & $1-3$ meses & $\begin{array}{l}\text { - Resecciones oncológicas y biopsias* } \\
\text { - Hernias } \\
\text { - Histerectomías } \\
\text { - Cirugía reconstructiva } \\
\text { - Cambios valvulares }\end{array}$ \\
\hline Cirugía opcional & $>3$ meses & $\begin{array}{l}\text { - Cirugía estética } \\
\text { - Cirugía bariátrica } \\
\text { - Procedimientos para infertilidad }\end{array}$ \\
\hline
\end{tabular}

Tabla de Stahel P. Modificada con procedimientos quirúrgicos clasificados por tiempo de espera para su realización. * Se debe individualizar a cada paciente. ${ }^{11}$ 
Tabla 2: Ventajas y desventajas del uso de la laparoscopía en tiempos de pandemia por COVID-19.

Ventajas

- Disminución del tiempo de estancia intrahospitalaria y posibles complicaciones

- En pacientes COVID-19 (+), al tratarse de un procedimiento menos traumático, pudiera resultar en un incremento en la supervivencia y recuperación

- La cavidad abdominal del paciente funciona como barrera física entre el cirujano y la posible fuente de contagio

- Permite una mayor distancia espacial entre paciente-cirujano, así como entre cirujanos
Desventajas

- El neumoperitoneo insuflado tiene una concentración de partículas elevada

- Posible incremento en el tiempo quirúrgico

- El uso de equipo específico para disminuir la fuga de neumoperitoneo aumenta costos y uso de recursos

- Mayor requerimiento del uso de energía avanzada

- El retiro de piezas quirúrgicas, trocares o neumoperitoneo pueden llegar a ocasionar lesiones que incrementen la morbilidad del procedimiento

- Resulta más complicada la filtración de partículas aerosolizadas en la cirugía abierta en comparación con la cirugía laparoscópica durante la pandemia, permitiendo tener una medida ética y moral al momento de la toma de decisiones. ${ }^{12}$

\section{Cirugía electiva}

Las cirugías electivas deberán cancelarse o postergarse considerando que esta medida aporta múltiples beneficios. Reduce la ocupación de camas hospitalarias y aumenta la capacidad para recibir pacientes con necesidad de atención médica por COVID-19. Permite disminuir el riesgo de exposición del personal quirúrgico, estando disponibles para nuevas asignaciones de actividades en relación al manejo de pacientes infectados. Disminuye el uso de insumos materiales y recursos económicos del hospital. ${ }^{8}$ Los beneficios de posponer una cirugía electiva también beneficiarán a los pacientes. El estrés metabólico posquirúrgico representa un factor de riesgo importante para inmunosupresión y predisposición a infección grave por COVID-19. ${ }^{13}$

\section{Cirugía laparoscópica}

El uso de cirugía laparoscópica ha sido un tema ampliamente discutido por los cirujanos a nivel mundial. Se ha demostrado que la vía de transmisión del SARS-CoV-2 es a través de gotas emitidas por vía respiratoria. Sin embargo, se ha detectado el virus en todo el tracto gastrointestinal, desde la boca hasta el recto, encontrando ácidos nucleicos virales en la saliva, bilis y heces de pacientes infectados. Se debe tener en mente la posibilidad de que el tracto digestivo pueda funcionar como una ruta alterna de infección. ${ }^{14-17}$ Además, la cirugía laparoscópica es considerada como un procedimiento generador de aerosoles. Aunque aún no contamos con evidencia científica que confirme esta posible vía de in- fección, ya se ha demostrado anteriormente la presencia de otros virus como el VHB en el neumoperitoneo. ${ }^{14,16-21}$ Las ventajas y desventajas del uso de la laparoscopía se enlistan en la Tabla 2.

\section{Recomendaciones para el abordaje laparoscópico:}

1. Disminuir el uso de energía avanzada: se ha estudiado la posibilidad de aerosolización de partículas con el uso de energía avanzada como lo es el electrocauterio monopolar, bipolar, láser o energía ultrasónica. ${ }^{14}$ Se deben colocar las unidades del aparato electroquirúrgico al mínimo necesario posible y disminuir el tiempo de disección. $7,19,20,24$ En caso de contar con uno, utilizar el lápiz monopolar con sistema de evacuación de humo incluido. ${ }^{19}$ Dejar como última posibilidad el uso de energía ultrasónica, ya que forma aerosoles de baja temperatura que pudiera no desactivar de manera efectiva los componentes celulares del virus..$^{20,24}$

2. Disminuir la presión de insuflación del neumoperitoneo y mantenerla al mínimo con el propósito de reducir el riesgo de aerosolizar el virus. $14,19,20,24$

3. Mantener en todo momento un circuito cerrado: se recomienda el intercambio mínimo de instrumentos durante el procedimiento, verificando su adecuado funcionamiento para disminuir el riesgo de fuga a través del trocar. ${ }^{14}$

a. Evacuación del neumoperitoneo: un punto de máxima importancia es el uso de sistemas de filtración y evacuación de gas y partículas, existen de tipo activo y pasivo. Estos sistemas logran filtrar con una eficiencia de hasta el $99.99 \%$ partículas iguales o mayores a 0.1 micras. ${ }^{17,20}$ Los activos 
$\left(\right.$ AirSeal $^{\odot}{ }^{\oplus}$ Pneumoclear ${ }^{\odot}$ ) son sistemas inteligentes cuyo puerto de acceso no cuenta con sistema de válvula y cuentan con la función conjunta de introducir y extraer el $\mathrm{CO}_{2}$ previa filtración del mismo. ${ }^{14,17}$ Los pasivos (SeeClear ${ }^{\odot}$ ) conectan el evacuador de humo al trocar menos utilizado, el cual filtra partículas a su extracción. ${ }^{17,24}$

Debido a que no todos los hospitales cuentan con la posibilidad para obtener los filtros, se desarrolló una medida simple y efectiva de evacuar el humo y neumoperitoneo. Para su realización, se debe conectar un tubo estándar al trocar de un extremo y al filtro incluido en los sistemas de ventilación mecánica mediante un conector de tubo endotraqueal, sin requerir succión activa para el proceso de filtración. ${ }^{21,23}$ Se debe evitar también la posible contaminación del sistema de insuflación, recomendando el cierre de la válvula del puerto de trabajo utilizado para la insuflación antes de detener el flujo de $\mathrm{CO}_{2}{ }^{17}$

b. Existen múltiples momentos de contacto con el neumoperitoneo durante el procedimiento. ${ }^{24}$ Hay que colocar el menor número de trocares posible y rea- lizar una incisión de menor tamaño que permita la entrada del trocar evitando la fuga. ${ }^{14,16,19}$ No se debe realizar la extracción intermitente de humo a través del trocar para mantener visibilidad del campo quirúrgico. ${ }^{14,17}$ Evitar la introducción de gasas laparoscópicas, disminuyendo la posibilidad de fuga de gas al momento de su introducción y extracción. ${ }^{14,24}$ La extracción de piezas quirúrgicas se debe realizar previa desinsuflación del neumoperitoneo en su totalidad. ${ }^{14,17}$ Intentar evacuar el humo en una sola ocasión. ${ }^{16}$ Se recomienda evitar el uso de cirugías mano-asistidas y sistemas de cierre de pared que permitan la fuga de gas. Se recomienda el cierre de la fascia posterior al retiro del neumoperitoneo. ${ }^{17,24}$

4. Evitar dentro de lo posible la posición de Trendelenburg para disminuir las repercusiones fisiológicas que tiene el neumoperitoneo sobre el sistema respiratorio y circulatorio..$^{20,24}$

5. El personal que se encuentre dentro del quirófano debe comunicarse de manera activa y clara sobre los pasos a realizar para disminuir el riesgo de accidentes. ${ }^{24}$

\section{Tabla 3: Patologías quirúrgicas de urgencia frecuentes y sus consideraciones especificas.}

Enfermedad hemorroidal

Abscesos perianales

y perirrectales

Infección de tejidos blandos

Infección de tejidos blandos

Neumoperitoneo, obstrucción o isquemia intestinal

Apendicitis

Patología biliar

Colecistitis aguda

Diverticulitis
Tratamiento quirúrgico de urgencia en caso de presentar sangrado, trombosis o fracaso de tratamiento médico

Realizar drenaje con incisión local. En caso de no contar con sala quirúrgica, considerar el realizar el abordaje por vía percutánea

Evaluar la extensión y localización de la infección para determinar si es necesario manejo en sala quirúrgica o con anestesia local, evaluar el riesgo de presentar fascitis necrotizante

Evaluar la extensión y localización de la infección para determinar si es necesario manejo en sala quirúrgica o con anestesia local, evaluar el riesgo de presentar fascitis necrotizante

Deberá realizarse el manejo conservador y evaluar las condiciones del paciente para realizar manejo quirúrgico de urgencia

Considerar manejo conservador para apendicitis no complicada (el tratamiento con antibióticos ha reportado un porcentaje de falla de 30-50\%). El abordaje laparoscópico ofrece baja morbilidad y menos días de estancia hospitalaria. En caso de apendicitis complicada el abordaje quirúrgico por vía laparoscópica es la mejor opción

En caso de colelitiasis sintomática, se deberá optar por el manejo farmacológico para control de síntomas y realizar programación quirúrgica de manera electiva. Si el paciente presenta coledocolitiasis que requiera CPRE, se sugiere realizar protocolo prequirúrgico para descartar infección por COVID-19

En pacientes sin comorbilidades o riesgo quirúrgico se sugiere resolución por vía laparoscópica. Si las condiciones del paciente no permiten el abordaje laparoscópico, se sugiere manejo con antibióticos vía IV y colecistostomía

Considerar la forma clínica de presentación; en caso de estadios clínicos según escala de Hinchey la o lb podrá realizarse el manejo con antibiótico vía IV, estadio clínico II y III considerar drenaje percutáneo y manejo antibiótico y estadios clínicos III o IV con falla al manejo conservador realizar abordaje quirúrgico 
Tabla 4: Fases de la pandemia COVID-19. Se recomienda realizar la cirugía sólo en patologías que pongan en riesgo la vida en el tiempo especificado en cada apartado.

\begin{tabular}{|c|c|c|}
\hline Fases & Clasificación & Tiempo \\
\hline \multirow[t]{3}{*}{ Fase aguda } & $\begin{array}{l}\text { Fase I: fase de preparación, se trabaja en un ambiente semiurgente, pocos pacientes con CO- } \\
\text { VID-19 y los recursos materiales aún disponibles }\end{array}$ & 1-3 meses \\
\hline & $\begin{array}{l}\text { Fase II: fase de rápida evolución, se trabaja en un ambiente urgente, con personal y recursos } \\
\text { limitados }\end{array}$ & Días \\
\hline & Fase III: los recursos materiales y físicos se encuentran agotados & Horas \\
\hline \multirow[t]{2}{*}{ Fase de recuperación } & $\begin{array}{l}\text { Fase temprana: posterior al pase del pico de la pandemia, con menor cantidad de casos cada } \\
\text { día y un incremento de recursos disponibles }\end{array}$ & Días \\
\hline & $\begin{array}{l}\text { Fase tardía: posterior al pase del pico de la pandemia con un mínimo de } 14 \text { días. Los recursos } \\
\text { casi llegan de nuevo a sus niveles normales }\end{array}$ & 1-3 meses \\
\hline
\end{tabular}

\section{Cirugía de urgencia}

En el caso de cirugía de urgencia, las recomendaciones se han centrado en enfocar los esfuerzos para brindar al paciente la atención quirúrgica oportuna, tomando en cuenta la seguridad tanto del paciente como del personal médico. La ACS ha descrito las patologías quirúrgicas más frecuentes y sus consideraciones en cuanto a criterios de urgencias (Tabla 3). ${ }^{25}$

\section{Consideraciones específicas por especialidad}

Todas las especialidades quirúrgicas se encuentran trabajando en sus propias recomendaciones con el propósito de disminuir al máximo las posibles repercusiones a lo largo de la pandemia. Se tienen que minimizar los procedimientos quirúrgicos sin comprometer los resultados. ${ }^{26}$ Dentro de lo posible, se debe optar por procedimientos de mínima invasión que acorten la estancia intrahospitalaria. ${ }^{27,28}$ Los servicios deben planear un programa de actividades y respuestas al término de la pandemia puesto que probablemente se enfrentarán a un mayor número de pacientes y estadios avanzados de enfermedades no atendidas en los últimos meses. ${ }^{27}$ El ACS realizó un sistema de clasificación de la pandemia en dos fases para orientar al cirujano en la realización de un triaje (Tabla 4). Algunas especialidades quirúrgicas lo han adaptado para sus patologías específicas. ${ }^{29}$

\section{Cirugía oncológica}

Los pacientes con diagnóstico de cáncer representan para el cirujano uno de los retos más grandes a enfrentar durante la pandemia, ya que son más susceptibles a infectarse por un estado de inmunosupresión sistémica. Liang y colaboradores estudiaron a 1,590 pacientes con COVID-19, enfocándose en la evolución de aquellos con cáncer (25\% bajo tratamiento dentro del último mes y $75 \%$ en periodo libre de enfermedad). Dentro de los resultados se detectó un riesgo 3.5 mayor de requerir ingreso a UTI, ventilación mecánica invasiva (VMI), de fallecer o presentar deterioro clínico y alargar la estancia intrahospitalaria (13 vs 43 días). ${ }^{30}$

Para lograr enfrentar este reto y continuar con la atención de los pacientes con cáncer de forma segura, se han propuesto múltiples estrategias. Se recomienda posponer visitas agendadas y realizar un triaje telefónico para consultar el estado clínico del paciente. ${ }^{31}$ No se debe permitir acceso a cuidadores, a menos que sea fundamental para el cuidado del paciente. ${ }^{30}$ Considerar la posibilidad de retrasar el tratamiento de manera individualizada. ${ }^{31}$ Establecer una vigilancia intensiva cuando se tiene a un paciente con cáncer infectado con COVID-19. ${ }^{30}$

¿Cómo tomar la decisión entre operar o esperar sin afectar la supervivencia? Para tomar esta decisión, también hay que considerar que la sinergia entre cáncer, cirugía y COVID-19 puede contribuir a una alta tasa de mortalidad (mortalidad perioperatoria de hasta el $20 \%$ ). $^{29}$ Y aunque la cirugía oncológica no suele ser considerada como electiva, en tiempos de pandemia se requiere identificar cuáles procedimientos son más urgentes que otros y valorar el riesgo-beneficio. ${ }^{29,32}$

El ACS realizó una guía con recomendaciones específicas para los principales tipos de cáncer. Esta guía tiene como intención el manejar y mitigar las posibles repercusiones del aplazamiento de las cirugías oncológicas, priorizando a los pacientes dependiendo de características específicas de su diagnóstico y proponiendo alternativas seguras basadas en la evidencia científica disponible. ${ }^{29}$ La Sociedad Quirúrgica Oncológica también emitió recomendaciones para el manejo de cada tipo de cáncer, asumiendo un retraso de entre tres y seis meses en la atención. ${ }^{33}$ 


\section{Cirugía endocrinológica}

La mayoría de las cirugías endocrinológicas pueden ser diferidas. Aquéllas que pueden clasificarse como urgentes y ser tratadas dentro de cuatro a ocho semanas se enlistan en la Tabla $5 \cdot 33,34$

\section{Cirugía cardiaca y torácica}

En el caso de pacientes con enfermedades cardiacas es necesario tomar en cuenta las necesidades y condiciones del paciente. Se deberán evaluar aquellos procedimientos que puedan hacerse por abordaje endovascular, ya que generan una vía de mínima invasión. ${ }^{35}$ Podemos dividir los procedimientos de cirugía torácica en tres fases acorde a la clasificación del ACS. En la fase I se deberán operar los nódulos pulmonares mayores de $2 \mathrm{~cm}$, tumores mediastinales sintomáticos, tumores de pared torácica, cáncer esofágico T1b o más. En la fase II se operarán las perforaciones secundarias a cáncer esofágico en paciente no séptico, tumor asociado a proceso infeccioso, complicaciones como hemotórax o empiema. Durante la fase III se operan solamente patologías que pongan en riesgo la

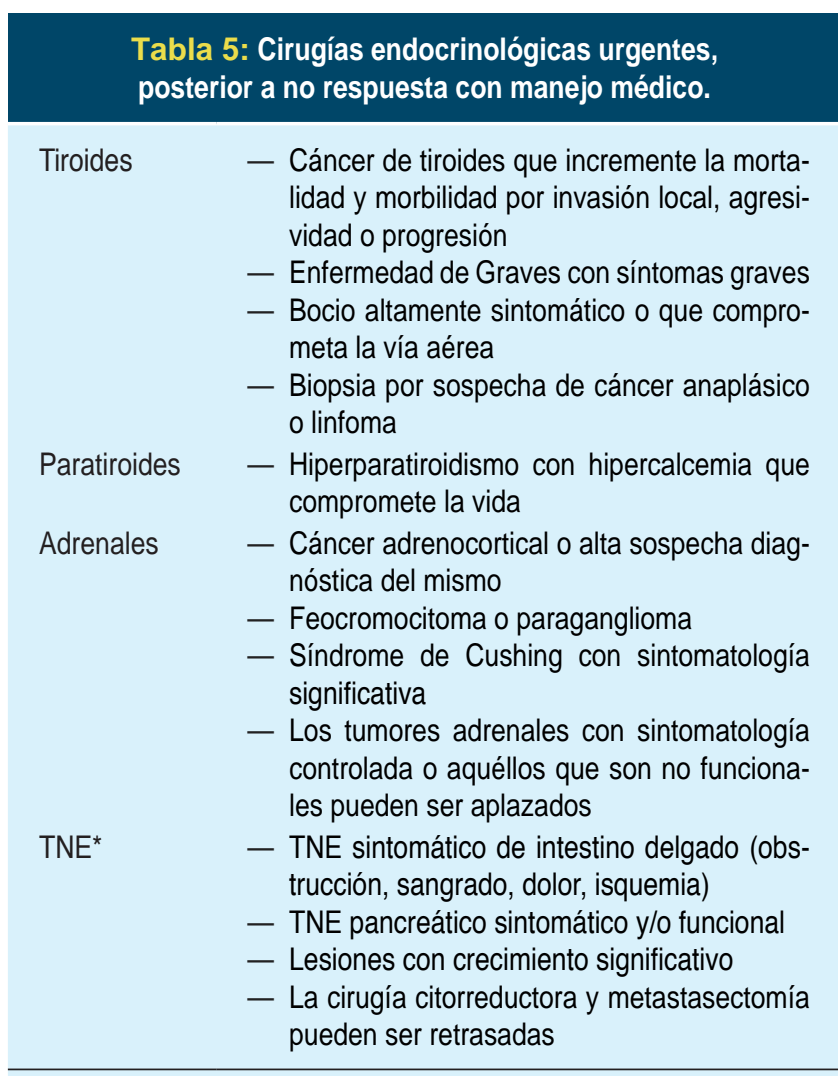

${ }^{*}$ TNE $=$ tumor neuroendocrino. ${ }^{33,34}$ vida en cuestión de horas, como la perforación esofágica asociado con sepsis, compromiso de vía aérea, hemotórax masivo o hemorragia activa. ${ }^{36}$

\section{Cirugía vascular}

Los casos de cirugía vascular deberán valorarse de manera individual de acuerdo a las condiciones del paciente y de la institución en que se encuentren. El Colegio Americano de Cirujanos ha propuesto guías para categorizar las condiciones quirúrgicas de cada probable padecimiento y cómo deben ser abordadas (Tabla 6). ${ }^{25}$ Usualmente, los pacientes del Servicio de Cirugía Vascular tienen alguna condición que pone en riesgo su vida o extremidades, por esto es importante continuar su seguimiento en clínicas o consulta externa. ${ }^{27}$

\section{Cirugía colorrectal}

Todo procedimiento colorrectal típicamente programado como de rutina debe ser diferido. Las patologías urgentes han sido descritas en el apartado de cirugía de urgencia de este artículo. Los casos que se deben tomar en cuenta son aquéllos que ponen en riesgo la vida del paciente en cuestión de horas como perforaciones, obstrucciones o sangrados activos. Las recomendaciones del ACS para patologías colorrectales se resumen en la Tabla $7 .{ }^{25}$ Todos los demás casos deben ser diferidos. Para la cirugía colorrectal de urgencia se recomienda la cirugía abierta en lugar de la laparoscópica para disminuir el riesgo de aerosolización. ${ }^{37}$ Como manejo alternativo, se puede considerar trasladar al paciente a algún centro con capacidad, la administración de tratamientos como radiación, quimioterapia neoadyuvante o realización de estomas derivativos.

\section{Cirugía bariátrica}

Las recomendaciones del ACS se resumen en la Tabla $8 .{ }^{25}$ Se sugiere que todos los procedimientos endoscópicos y quirúrgicos de cirugía bariátrica sean pospuestos durante la pandemia. ${ }^{38}$ Sólo emergencias relacionadas a complicaciones posoperatorias de cirugía bariátrica deberán atenderse de manera inmediata. Los pacientes de clínicas de obesidad y bariatría representan una población en riesgo para estrés emocional asociado a desórdenes alimentarios que podrían verse exacerbados durante la pandemia. ${ }^{39}$ Esto confiere un aumento de riesgo para complicaciones metabólicas y riesgo de presentar una infección severa por COVID-19. ${ }^{40}$ Se recomienda su seguimiento por medio de consultas virtuales para disminuir el riesgo de aumento de peso y continuar los programas de modificación del estilo de vida. ${ }^{39}$ 


\section{Tabla 6: Nivel de atención para cada condición de cirugía vascular (*REF ACS).}

\begin{tabular}{|c|c|}
\hline Nivel de atención & Condiciones \\
\hline $\begin{array}{l}\text { No posponer cirugía: } \\
\text { emergencia quirúrgica }\end{array}$ & $\begin{array}{l}\text { - AAA/AATA. Roto o sintomático } \\
\text { - Aneurisma asociado con infección o infección de material protésico } \\
\text { - Aneurisma periférico sintomático } \\
\text { - Pseudoaneurisma periférico complejo, expansivo, no candidato a manejo con trombina } \\
\text { - Aneurisma abdominal no aórtico sintomático } \\
\text { - Disección aórtica con ruptura o malperfusión } \\
\text { - Fístula aorto-esofágica con choque séptico o choque hemorrágico } \\
\text { - Prótesis arterial infectada con signos de choque séptico, choque hemorrágico o riesgo alto de ruptura } \\
\text { - Estenosis carotídea sintomática } \\
\text { - Acceso para hemodiálisis disfuncional/trombosado/infectado } \\
\text { - FAV con ulceración } \\
\text { - Falla renal con necesidad de acceso para hemodiálisis } \\
\text { - Acceso para hemodiálisis tunelizado } \\
\text { - Trombosis mesentérica aguda sintomática } \\
\text { - Isquemia aguda de extremidades/gangrena húmeda/celulitis ascendente } \\
\text { - Síndrome compartimental con necesidad de fasciotomía } \\
\text { - Lesión vascular traumática con hemorragia o isquemia } \\
\text { - TVP iliofemoral aguda con flegmasía } \\
\text { - Amputaciones por infección/necrosis } \\
\text { - Cirugía/embolización para sangrado no controlable en pacientes inestables }\end{array}$ \\
\hline $\begin{array}{l}\text { Valorar posponer cirugía: } \\
\text { Manejo médico } \\
\text { y vigilancia estrecha }\end{array}$ & $\begin{array}{l}\text { - AAA/AATA > } 6.5 \mathrm{~cm} \\
\text { - Aneurisma periférico asintomático } \\
\text { - Aneurisma abdominal no aórtico sintomático } \\
\text { - Estenosis de alto grado de intervenciones previas } \\
\text { - Estenosis carotídea asintomática } \\
\text { - Revisión de FAV/fistulografía por disfunción } \\
\text { - Creación de FAV } \\
\text { - Isquemia mesentérica crónica } \\
\text { - Isquemia crónica de extremidades (dolor en reposo) } \\
\text { - Trombólisis arterial o venosa } \\
\text { - SST venoso sintomático con oclusión aguda y edema } \\
\text { - SST arterial con trombosis } \\
\text { - Colocación de FVC } \\
\text { - TVP iliofemoral sintomática en pacientes de bajo riesgo } \\
\text { - Úlceras secundarias a enfermedad venosa } \\
\text { - Lavado quirúrgico/ debridación de heridas quirúrgicas o necrosis } \\
\text { - Exposición para abordaje anterior de instrumentación lumbar } \\
\text { - Cirugía/embolización para sangrado no controlable en pacientes estables } \\
\text { - Colocación de catéter puerto para medicamentos } \\
\text { - Retiro de catéter puerto por complicaciones }\end{array}$ \\
\hline $\begin{array}{l}\text { Posponer cirugía: } \\
\text { manejo médico y vigilancia }\end{array}$ & $\begin{array}{l}\text { - AAA }<6.5 \mathrm{~cm} \\
\text { - Estenosis asintomática de injertos/stents } \\
\text { - Claudicación } \\
\text { - SST neurogénico } \\
\text { - May-Thurner asintomático } \\
\text { - Retiro de FVC } \\
\text { - Safenectomías }\end{array}$ \\
\hline
\end{tabular}

*AAA = Aneurisma aórtico abdominal, AATA = Aneurisma aórtico toracoabdominal, FAV = fístula arteriovenosa, TVP = trombosis venosa profunda, FVC = filtro de vena cava, SST = síndrome salida torácica. ${ }^{25}$ 
Tabla 7: Nivel de atención para patologías colorrectales.

\begin{tabular}{|c|c|}
\hline Nivel de atención & Condiciones \\
\hline $\begin{array}{l}\text { Emergencia: } \\
\text { atención inmediata }\end{array}$ & $\begin{array}{l}\text { - Obstrucción intestinal } \\
\text { - Perforación intestinal } \\
\text { - Sangrados activos } \\
\text { - Vólvulo colónico* } \\
\text { - Absceso perianal o perirrectal y fístu- } \\
\text { la compleja } \\
\text { - Diverticulitis aguda Hinchey III-IV }\end{array}$ \\
\hline $\begin{array}{l}\text { Urgencia: } \\
\text { necesita cirugía, } \\
\text { puede posponerse } \\
\text { días o semanas }\end{array}$ & $\begin{array}{l}\text { - Tumor colorrectal suboclusivo* } \\
\text { - Cáncer que requiere transfusiones } \\
\text { sanguíneas frecuentes } \\
\text { - Cáncer rectal asintomático } \\
\text { - Cáncer que ha recibido tratamiento } \\
\text { neoadyuvante } \\
\text { - Tumores con riesgo de perforación o } \\
\text { sepsis } \\
\text { - Diverticulitis aguda recurrente o } \\
\text { Hinchey Ib-II* } \\
\text { - Prolapso rectal o rectocele recurrente* } \\
\text { - Fístula anal } \\
\text { - Enfermedad pilonidal } \\
\text { - Hidrosadenitis supurativa* } \\
\text { - Enfermedad hemorroidal grado III-IV* }\end{array}$ \\
\hline $\begin{array}{l}\text { Electiva: } \\
\text { puede ser pospuesta } \\
\text { hasta por 3-6 meses } \\
\text { sin riesgo }\end{array}$ & $\begin{array}{l}\text { - Enfermedad hemorroidal grado } \|-I^{*}{ }^{*} \\
\text { - Pólipos malignos o de características } \\
\text { sospechosas } \\
\text { - Procedimientos profilácticos en condi- } \\
\text { ciones hereditarias } \\
\text { - Carcinoma colorrectal in situ } \\
\text { - Enfermedad inflamatoria intestinal } \\
\text { recurrente }\end{array}$ \\
\hline
\end{tabular}

* Se debe de considerar el manejo quirúrgico en caso de que no se haya obtenido una adecuada respuesta al tratamiento conservador 0 intervencionista. ${ }^{25}$

\section{Cirugía de trasplantes}

En pacientes receptores de trasplantes existe un riesgo incrementado de infección al presentar un estado de inmunosupresión continuo. Aún no existen estudios que demuestren el riesgo de pacientes receptores de trasplantes de contraer infección por COVID-19. Basado en el comportamiento de otros virus, las infecciones suelen ser más severas en este grupo de pacientes..$^{41} \mathrm{El}$ riesgo de contagio por injertos es bajo, aunque siempre se debe realizar un tamizaje preoperatorio para COVID-19. Si se tiene planeado un trasplante, es necesario diferirlo de 14 a 28 días si el receptor o donador visitó o vive en un área de alta prevalencia. ${ }^{41} \mathrm{La} C \mathrm{CD}$ recomienda no posponer cirugías de trasplantes en pacientes con alto riesgo de complicaciones o mal estado de salud.
Se han tenido reportes de pacientes bajo terapia inmunosupresora con potencial para falsos negativos en pruebas para COVID-19, aún no existe evidencia para realizar recomendaciones. ${ }^{42}$ Se debe continuar terapia con antihipertensivos aunque existe evidencia de que la sobreexpresión de receptores de angiotensina II secundario al uso de éstos. La terapia inmunosupresora aumenta el riesgo de desarrollar enfermedad severa en recipientes de trasplantes, pero no se recomienda suspender o disminuir la dosis. ${ }^{42}$

\section{Cirugía plástica y reconstructiva}

La Asociación Americana de Cirugía Plástica recomienda que se suspendan todas las cirugías electivas y no esenciales. ${ }^{25}$ Las cirugías reconstructivas electivas urgentes como cobertura cutánea con colgajos en heridas crónicas o colocación de injertos pueden ser diferidas a criterio del cirujano. Se recomienda que sólo se realicen cirugías reconstructivas urgentes, incluyendo aquéllas relacionadas con pacientes quemados y reconstrucciones de lesiones que impliquen alguna afección vásculo-nerviosa que ponga en peligro la función. ${ }^{43}$

\section{Neurocirugía}

Procedimientos como traumatismos e infecciones, ya sean abscesos, empiemas o fracturas, requieren cirugía en $<$

\begin{tabular}{|c|c|}
\hline \multicolumn{2}{|c|}{$\begin{array}{l}\text { Tabla } 8 \text { : Nivel de atención para condiciones } \\
\text { bariátricas y su manejo quirúrgico. }\end{array}$} \\
\hline Nivel de atención & Condiciones \\
\hline $\begin{array}{l}\text { Emergencia: } \\
\text { atención inmediata }\end{array}$ & $\begin{array}{l}\text { - Úlcera marginal perforada } \\
\text { - Sangrado de anastomosis } \\
\text { - Fuga de anastomosis } \\
\text { - Obstrucción por hernia interna } \\
\text { - Perforación de banda gástrica } \\
\text { - Prolapso de banda gástrica }\end{array}$ \\
\hline $\begin{array}{l}\text { Urgencia: } \\
\text { necesita cirugía, } \\
\text { puede posponerse } \\
\text { días o semanas }\end{array}$ & $\begin{array}{l}\text { - Revisión por disfagia } \\
\text { - ERGE severo } \\
\text { - Deslizamiento de banda gástrica } \\
\text { - Estenosis en anastomosis con riesgo } \\
\text { de aspiración }\end{array}$ \\
\hline $\begin{array}{l}\text { Electiva: } \\
\text { puede } \\
\text { ser pospuesta } \\
\text { por meses } \\
\text { sin riesgo }\end{array}$ & $\begin{array}{l}\text { - Derivación gastroyeyunal } \\
\text { - Manga gástrica } \\
\text { - Banda gástrica } \\
\text { - Derivación biliopancreática con cruce } \\
\text { duodenal }\end{array}$ \\
\hline
\end{tabular}

ERGE = enfermedad por reflujo gastroesofágico.$^{25}$ 
48 horas. Los tumores que producen efecto de masa y la apoplejía pituitaria también son casos urgentes. Dentro de este rubro se incluye cualquier patología que ponga en peligro la médula espinal, ya sea por fracturas, tumores 0 infecciones, o por síndromes como el de cauda equina que merman la calidad de vida del paciente de forma importante y permanente. La disfunción de válvulas o prótesis también requiere manejo urgente. El resto de los procedimientos se deben tratar de trasladar o diferir dependiendo del nivel de urgencia. ${ }^{44}$

\section{Urología}

La Asociación Europea de Urología publicó recomendaciones preliminares para el adecuado triage de las patologías urológicas. ${ }^{26} \mathrm{~A}$ su vez, el Departamento de Urología de la Clínica de Cleveland creó un sistema de estratificación, el cual clasifica las patologías y los procedimientos ambulatorios acorde a su grado de urgencia. ${ }^{45}$ Las patologías oncológicas tienen una alta incidencia (cáncer de próstata 7.1\%, vejiga $3 \%$, renal $2.2 \%$ ), Ficarra y colaboradores clasificaron los distintos tipos de cáncer y estadios en cuatro grupos (no diferible, semi-no diferible, diferible y reemplazable) para orientar el manejo adecuado. ${ }^{28,46}$ Las patologías benignas como incontinencia, hiperplasia prostática benigna, cirugías reconstructivas, infertilidad, disfunción eréctil y prolapso genitourinario deben ser diferidas posterior a la finalización de la pandemia. ${ }^{28,46}$ Las urgencias que ponen en peligro la vida como obstrucción e infección del tracto urinario superior, retención urinaria aguda, retención de coágulo, trauma genitourinario, torsión de cordón espermático, infección de prótesis, absceso escrotal y priapismo deben ser atendidas en cuestión de horas. ${ }^{28,46}$

\section{Cirugía pediátrica}

En cirugía pediátrica, aparte de las patologías quirúrgicas de urgencia como la apendicitis aguda, torsión ovárica o testicular, atresia esofágica o intestinal, se considera que las cirugías oncológicas, gastrostomías, accesos vasculares y hernias inguinales sintomáticas no deberán retrasarse. ${ }^{47}$

\section{Otorrinolaringología}

La nasolaringoscopia, la intubación endotraqueal, traqueostomías, la cirugía endoscópica transnasal, instrumentos ultrasónicos son procedimientos que aumentan el riesgo de contagio al personal de salud. Los cirujanos que realizan cirugía endoscópica de senos paranasales tienen un riesgo aumentado en comparación con otros procedimientos endoscópicos por la alta carga viral encontrada en la nasofaringe de pacientes infectados. ${ }^{48}$ La Academia Americana de Otorrinolaringología recomienda que se realicen sólo procedimientos urgentes y propone una lista de procedimientos que no se deben diferir. ${ }^{48}$

\section{Complicaciones y evolución posquirúrgica}

Existe un estudio de cohorte realizado en la ciudad de Wuhan en donde se revisó de manera retrospectiva a pacientes que se encontraban en periodo de incubación de COVID-19 y en los cuales se llevó a cabo cirugía electiva documentando la evolución posquirúrgica. Se reportó que el $44.1 \%$ requirieron admisión a la UTI, y $20 \%$ de éstos falleció. Dentro de las complicaciones posquirúrgicas presentaron falla respiratoria, choque, arritmia, lesión cardiaca aguda y lesión renal aguda. ${ }^{49}$ Para pacientes de alto riesgo que desarrollen fiebre o tos posterior a la cirugía se requiere una TC de tórax y una PCR. Se sabe que existe un mayor riesgo de trombosis venosa profunda, por lo que se debe dar tromboprofiláxis adecuada. ${ }^{49}$

En España se realizó un estudio observacional en el cual se intervinieron de manera urgente a 36 pacientes COVID-19 positivos, tres de ellos fallecieron en el periodo posquirúrgico. De todos los pacientes operados, $42 \%$ presentaron síntomas respiratorios sugestivos de la enfermedad..$^{50}$ Es importante tomar en cuenta que el paciente puede requerir en algún punto soporte ventilatorio o manejo en UTI. Se sugiere que los pacientes con adecuada evolución posquirúrgica se mantengan en un cuarto de aislamiento con vigilancia estrecha. Los criterios de alta dependen de la evolución del paciente, contar con dos pruebas consecutivas de PCR negativas, seguido de aislamiento preventivo en domicilio de siete días más.

\section{Indicaciones para el cuidado ambulatorio del paciente}

Con el propósito de disminuir el riesgo de infección en aquellos pacientes sanos y disminuir el uso de recursos, si el paciente no cuenta con alguna contraindicación que requiera su manejo intrahospitalario lo ideal será reducir al máximo su estancia y otorgar el seguimiento posoperatorio de manera ambulatoria. La telemedicina se ha convertido en una herramienta valiosa para mantener el seguimiento de los pacientes y una relación médico-paciente a distancia. El cirujano deberá prever la necesidad de evitar el contacto posterior, por lo cual se recomienda el cierre de heridas con uso de sutura subcuticular con sutura absorbible para evitar el retiro de puntos. ${ }^{24}$ Será de vital importancia comunicarles a los pacientes todos los posibles datos de alarma que puedan presentarse en caso de cuadro de COVID-19 o por el procedimiento quirúrgico realizado. En el caso de los pacientes con infección por COVID-19 que seguirán 
su manejo posoperatorio en casa, es importante explicarles las medidas y precauciones a seguir dictadas por la Organización Mundial de la Salud para evitar el contagio de sus contactos. ${ }^{8}$

\section{RESULTADOS}

Se obtuvo información actualizada sobre el manejo quirúrgico seguro durante la pandemia por COVID-19, basado en artículos emitidos por organizaciones internacionales de salud, manuales de manejo estandarizados, opiniones de expertos en el tema y por experiencias de otros países más avanzados en la pandemia. Se logró crear una guía de indicaciones quirúrgicas abarcando múltiples áreas y especialidades de interés que ayudarán al cirujano a llevar su práctica quirúrgica con seguridad durante y después de la pandemia.

\section{DISCUSIÓN}

En el ámbito mundial, han surgido múltiples dudas entre los cirujanos sobre la necesidad de diferir las cirugías electivas, el riesgo de aerosolización durante procedimientos laparoscópicos, el potencial riesgo que tiene el paciente de complicarse en el posoperatorio en caso de infectarse con COVID-19 durante su hospitalización, entre otras. Es importante aclarar que el término «cirugía electiva» no significa cirugía opcional, sino que se refiere a aquella cirugía que no está indicada inmediatamente para tratar algún padecimiento quirúrgico urgente o que ponga en riesgo la vida del paciente. Un alto porcentaje de las cirugías electivas tienen un daño potencial en el paciente al ser canceladas o retrasadas, por lo que cada caso debe ser individualizado.

En la cirugía de urgencia, las recomendaciones se han centrado en enfocar los esfuerzos para brindar al paciente la atención quirúrgica oportuna, sin olvidar la seguridad tanto del paciente como del personal médico. El EPP es siempre la principal prioridad durante cualquier procedimiento, incluso en casos de emergencia. ${ }^{9}$ Todo paciente que llegue al Servicio de Urgencias o que sea sometido a un procedimiento quirúrgico se tratará como un potencial caso sospechoso hasta que se pruebe lo contrario. ${ }^{6}$

Las diferentes especialidades quirúrgicas se encuentran trabajando en sus propias recomendaciones con el propósito de disminuir al máximo las posibles repercusiones. El ACS ha desarrollado un sistema de clasificación por fases, durante y después de la pandemia que puede ser adaptado a diferentes especialidades. ${ }^{29}$ Es importante recalcar que, al tratarse únicamente de recomendaciones emitidas por expertos y basadas en experiencias de otros países, el cirujano individualizará cada decisión, manteniendo siempre una adecuada comunicación médico-paciente. Debe exis- tir una toma de decisión conjunta revisada por un equipo multidisciplinario en la que todos los puntos relevantes fueron puestos en una balanza. ${ }^{29}$ Cada comunidad y ambiente es diferente y las recomendaciones establecidas varían en cuanto a las necesidades de cada región; por lo que la disponibilidad de recursos económicos, humanos y materiales también son considerados en la práctica clínica.

\section{CONCLUSIÓN}

La práctica quirúrgica como la conocemos ha cambiado radicalmente a raíz de la pandemia. La información recabada de la literatura actual disponible únicamente realiza recomendaciones que surgen de datos ya conocidos y de opiniones de expertos. Esta guía de recomendaciones no debe ser seguida rigurosamente, es necesario aplicar el criterio del cirujano en cada situación específica. Situaciones no modificables como la escasez de recursos y el riesgo-beneficio de que el paciente reciba un tratamiento bajo la infección con COVID-19 o el riesgo de contraerla, obligan al cirujano a adaptarse a las circunstancias. Existe incertidumbre en cuanto a la evolución de la pandemia y por esto nuevos hallazgos hacen que la información cambie rápidamente. Es probable que las estrategias y medidas descritas en este documento cambien conforme vamos adquiriendo conocimiento sobre el COVID-19.

\section{REFERENCIAS}

1. Coronaviridae Study Group of the International Committee on Taxonomy of Viruses. The species severe acute respiratory syndrome-related coronavirus: classifying 2019-nCoV and naming it SARS CoV-2. Nat Microbiol. 2020; 5: 536-544.

2. Ren LL, Wang YM, Wu ZQ, Xiang ZC, Guo L, Xu T et al. Identification of a novel coronavirus causing severe pneumonia in human: a descriptive study. Chin Med J (Engl). 2020; 133: 1015-1024.

3. Yang W, Wang C, Shikora S, Kow L. Recommendations for Metabolic and Bariatric Surgery During the COVID-19 Pandemic from IFSO. Obes Surg. 2020; 30: 2071-2073.

4. Zu ZY, Jiang MD, Xu PP, Chen W, Ni QQ, Lu GM, Zhang LJ.Coronavirus disease 2019 (COVID-19): a perspective from China. Radiology. 2020; 200490.

5. Coimbra R, Edwards S, Kurihara H, Bass GA Balogh ZJ, Tilsed $\mathrm{J}$ et al. European Society of Trauma and Emergency Surgery (ESTES) recommendations for trauma and emergency surgery preparation during times of COVID-19 infection. Eur J Trauma Emerg Surg. 2020: 1-6.

6. Coccolini F, Perrone G, Chiarugi M, Di Marzo F, Ansaloni L, Scandroglio I et al. Surgery in COVID-19 patients: operational directives. World J Emerg Surg. 2020; 15: 25.

7. Ti LK, Ang LS, Foong TW, Ng BSW. What we do when a COVID-19 patient needs an operation: operating room preparation and guidance. Can J Anesth. 2020; 67: 756-758. 
8. COVIDSurg Collaborative. Global guidance for surgical care during COVID-19 pandemic. Br J Surg. 2020.

9. American College of Surgeons. COVID-19: Considerations for optimum surgeon protection. 2020. https://www.facs. org/covid-19/clinical-guidance/surgeon-protection.

10. Wong J, Goh QY. Preparing for a COVID-19 pandemic: a review of operating room outbreak response measures in a large tertiary hospital in Singapore. Can J Anesth. 2020; 67: 732-745.

11. Stahel P. How to risk-stratify elective surgery during the COVID-19 pandemic? BMC. 2020; 14: 8.

12. Prachand VN, Milner R, Angelos P, Posner MC, Fung JJ, Agrawal $\mathrm{N}$ et al. Medically necessary, time-sensitive: Scoring system to ethically and efficiently manage resource scarcity and provider risk during the COVID-19 pandemic. J Am Coll Surg. 2020; S1072-7515(20)30317-3.

13. Besnier E, Tuech JJ, Schwarz L. We Asked the Experts: Covid-19 Outbreak: is there still a place for scheduled surgery? "Reflection from pathophysiological data". World J Surg. 2020; 44: 1695-1698.

14. Vigneswaran Y, Prachand VN, Posner MC, Matthews JB, Hussain M. What is the appropriate use of laparoscopy over open procedures in the current COVID-19 climate? J Gastrointest Surg. 2020: 1-6.

15. Gu J, Han B, Wang J. COVID-19: Gastrointestinal manifestations and potential fecal-oral transmission. Gastroenterology. 2020; 158: 1518-1519.

16. Balibrea JM, Badia JM, Rubio I, Martín E, Álvarez E, García $S$ et al. Manejo quirúrgico de pacientes con infección por COVID-19. Recomendaciones de la Asociación Española de Cirujanos. Cir Esp. 2020; 98 (5): 251-259.

17. SAGES Webmaster. Resources for smoke \& gas evacuation during open, laparoscopic, and endoscopic procedures. 2020. https://www.sages.org/resources-smoke-gasevacuation-during-open-laparoscopic-endoscopicprocedures/.

18. Brat GA, Hersey SP, Chhabra K, Gupta A, Scott J. Protecting surgical teams during the COVID-19 outbreak: a narrative review and clinical considerations. Annals of Surgery. 2020; 1-9.

19. Pryor A. SAGES and EAES recommendations regarding surgical response to COVID-19 crisis. 2020. https://www. sages.org/recommendations-surgical-response-covid-19/.

20. Zheng $\mathrm{MH}$, Boni L, Fingerhut A. Minimally invasive surgery and the novel coronavirus outbreak: lessons learned in China and Italy. Ann Surg. 2020; 10.1097/ SLA.0000000000003924.

21. Mintz Y, Arezzo A, Boni L, Chand M, Brodie R, Fingerhut A. A low cost, safe and effective method for smoke evacuation in laparoscopic surgery for suspected coronavirus patients. Ann Surg. 2020.

22. Brücher B, Nigri G, Tinelli A, Lapeña JFF, Espin-Basany E, Macri $P$ et al. COVID-19: Pandemic surgery guidance. B.L.D.M. 4 open. 2020; 3: 1-19.

23. Di Saverio S, Khan M, Pata F, Letto G, DeSimone B, Zani E et al. Laparoscopy at all costs? Not now during COVID-19 and not for acute care surgery and emergency colorectal surgery: a practical algorithm from a Hub Tertiary teaching hospital in Northern Lombardy, Italy. J Trauma Acute Care Surg. 2020.

24. UNMC Nebraska Medicine. Laparoscopy Adjustments During COVID-19 Addendum B. 2020. https://www. nebraskamed.com/sites/default/files/documents/covid-19/ laparoscopy-covid-19.pdf.

25. American College of Surgeons. COVID-19: Elective case triage guidance for surgical care. 2020. https://www.facs. org/covid-19/clinical-guidance/elective-case.

26. Stensland KD, Morgan TM, Moinzadeh A, Lee CT, Briganti A, Catto JWF, Canes D. Considerations in the triage of urologic surgeries during the COVID-19 pandemic. Eur Urol. 2020; S0302-2838(20)30202-5.

27. Gouveia E, Melo R, Pedro LM. Vascular surgery department adjustments in the era of the COVID-19 pandemic. J Vasc Surg. 2020; S0741-5214(20)30593-0.

28. Ficarra V, Novara G, Abrate A, Bartoletti R, Crestani A, De Nunzio $C$ et al. Urology practice during COVID-19 pandemic. Minerva Urol Nefrol. 2020.

29. American College of Surgeons. ACS Guidelines for Triage and Management of Elective Cancer Surgery Cases During the Acute and Recovery Phases of Coronavirus Disease 2019 (COVID-19) Pandemic. American College of Surgeons. 2020. Available in: https://www.facs.org/-/media/files/ covid19/acs_triage_and_management_elective_cancer surgery_during_acute_and_recovery_phases.ashx

30. Liang W, Guan W, Wang W, Li J, Xu K, Li C et al. Cancer patients in SARS-CoV-2 infection: a nationwide analysis in China. Lancet Oncol. 2020; 21: 335-337.

31. Lambertini M, Toss A, Passaro A, Criscitiello C, Cardone C, Loupakis F et al. Cancer care during the spread of coronavirus Disease 2019 (COVID-19) in Italy: young oncologists perspective. ESMO Open. 2020; 5: e000759.

32. Ueda M, Martins R, Hendrie PC, McDonnell T, Crews JR, Wong TL et al. Managing cancer care during the COVID-19 pandemic: agility and collaboration toward a common goal. J Natl Compr Canc Netw. 2020; 1-4.

33. Bartlett DL, Howe JR, Chang G, Crago A, Hogg M, Karakousis $G$ et al. Management of cancer surgery cases during the COVID-19 pandemic: considerations. Ann Surg Oncol. 2020; 27: 1717-1720.

34. Jozaghi Y, Zafereo ME, Perrier ND, Wang JR, Grubbs E, Gross ND et al. Endocrine Surgery in the Coronavirus Disease 2019 Pandemic. Head \& Neck. 2020: 1-10.

35. Mavioglu HL, Ertekin UU. Cardiovascular surgery in the COVID?19 pandemic. J Card Surg. 2020. https:// onlinelibrary.wiley.com/doi/full/10.1111/jocs.14559.

36. American College of Surgeons COVID 19: elective case triage guidelines for surgical care. Thoracic Cancer Surgery. 2020. https://www.facs.org/covid-19/clinical-guidance/ elective-case/thoracic-cancer.

37. Angelos G, Grayson A. Gachabayov M, Latifi R, Bergamaschi R. Emergency colorectal surgery in a COVID-19 pandemic epicenter. Surg Technol Int. 2020.

38. Yang W, Wang C, Shikora S, Kow L. Recommendations for Metabolic and Bariatric Surgery During the COVID-19 Pandemic from IFSO. Obes Surg. 2020; 1-3. 
39. Sockalingam S, Leung SE, Cassin SE. The Impact of COVID?19 on bariatric surgery: re? defining psychosocial care. Obesity. 2020.

40. Sattar N, Mclnnes IB, McMurray JJV. Obesity a risk factor for severe COVID-19 infection: multiple potential mechanisms. Circulation. 2020.

41. American Society of Transplantation. COVID19 resources for transplant community. 2020. https://www.myast.org/ covid-19-information\#.

42. National Kidney Foundation. Transplant \& COVID-19. 2020. https://www.kidney.org/coronavirus/transplantcoronavirus.

43. Jeffers L. American Society of Plastic Surgeons. COVID-19 statements: ASPS guidance regarding elective and nonessential patient care. 2020. https://www. plasticsurgery. org/for-medical-professionals/covid19-member-resources/ previous-statements.

44. Burke JF, Chan AK, Mummaneni V, Chou D, Lobo EP, Berger MS et al.. The coronavirus disease 2019 global pandemic: a neurosurgical treatment algorithm. Neurosurgery. 2020.

45. Goldman HB, Haber GP. Recommendations for tiered stratification of urological surgery urgency in the COVID-19 Era. J Urol. 2020.
46. Puliatti S, Eissa A, Eissa R, Amato M, Mazzone E, Dell'Oglio $P$ et al. COVID-19 and Urology: A Comprehensive Review of the Literature. BJU Int. 2020.

47. American College of Surgeons. COVID 19: elective case triage guidelines for surgical care: Pediatric surgery. 2020. https:// www.facs.org/covid-19/clinical-guidance/elective-case.

48. Kowalski LP, Sanabria A, Ridge JA, Ng WT, de Bree R, Rinaldo A et al. COVID-19 pandemic: Effects and evidencebased recommendations for otolaryngology and head and neck surgery practice. Head Neck. 2020.

49. Lei S, Jiang F, Su W, Chen C, Chen J, Mei W et al. Clinical characteristics and outcomes of patients undergoing surgeries during the incubation period of COVID-19 infection. E Clinical Medicine. 2020; 100331.

50. Álvarez M, Gortázar S, Pascual I, Rubio-Pérez I, Barragán C, Álvarez E. Díaz J. Impacto de la pandemia por SARS-CoV-2 sobre la actividad y profesionales de un Servicio de Cirugía General y del Aparato Digestivo en un hospital terciario. Cir Esp 2020;4(1).

Conflicto de intereses: Los autores declaran no tener ningún interés relacionado con el contenido de este artículo. 